\title{
Nitrogen abundance in turn-off stars of NGC 6397 and NGC $6752^{\star}$
}

\author{
L. Pasquini ${ }^{1}$, A. Ecuvillon ${ }^{2}$, P. Bonifacio ${ }^{3,4,5}$, and B. Wolff ${ }^{1}$
}

1 European Southern Observatory, Garching bei München, Germany

e-mail: lpasquin@eso.org

2 Institut d'Astrophysique, Paris, France

3 CIFIST Marie Curie Excellence Team, France

${ }^{4}$ GEPI, Observatoire de Paris, CNRS, Université Paris Diderot, Place Jules Janssen, 92190 Meudon, France

5 Istituto Nazionale di Astrofisica - Osservatorio Astronomico di Trieste, via Tiepolo 11, 34131 Trieste, Italy

Received 14 April 2008 / Accepted 12 June 2008

\section{ABSTRACT}

\begin{abstract}
Aims. Our goal is to measure the nitrogen abundance in 5 turn off (TO) stars of the two globular clusters NGC 6397 and NGC 6752 , and to compare the cluster abundances with those of field stars of comparable metallicity. In addition, we investigate the variations in nitrogen abundance and its connections to variations in the abundances of other light elements.

Methods. We determine the nitrogen abundance from the compact band-head of the (0-0) vibrational band of the $\mathrm{NH} A^{3} \Pi-X^{3} \Sigma^{-}$ system at $3360 \AA$, using spectra of resolution $R \sim 45000$ acquired using the UVES spectrograph on the ESO Kueyen-VLT $8.2 \mathrm{~m}$ telescope, analysed with spectrum synthesis based on plane parallel LTE model atmospheres. We apply a method previously applied to field stars, to enable a direct comparison to be made between the results.

Results. The nitrogen abundances of two stars in NGC 6397 are found to be almost identical, even though there is a difference of one order of magnitude in their oxygen abundances. In a third star of the same cluster, the nitrogen abundance is slighty lower, although consistent, within the calculated margins of error, with the other two measurements. All stars in NGC 6397 are N-rich with respect to field objects of similar metallicity. The two stars in NGC 6752 show a difference in nitrogen abundance of over one order of magnitude. The same stars differ in the abundances of other elements such as $\mathrm{Na}, \mathrm{O}$, and $\mathrm{Li}$, although only by a factor $\sim 3-4$.

Conclusions. NGC 6397 and NGC 6752 are, at present, the only two globular clusters in which N has been measured in TO stars in a way consistent with similar measurements for field stars. The behaviour of $\mathrm{N}$ is different in the two clusters: no variation is observed between stars in NGC 6397, while a large variation is observed in NGC 6752. This is consistent with a picture in which the stars in NGC 6752 have been formed by a mixture of "pristine" material and material which has been processed by an early generation of stars, referred to as "polluters". The $\mathrm{N}$ abundances reported will help to constrain the properties of the polluters. In the case of NGC 6397, a simple pollution history is probably not viable, since the observed variations in $\mathrm{O}$ abundances are not accompanied by corresponding variations in $\mathrm{N}$ or $\mathrm{Li}$.
\end{abstract}

Key words. stars: abundances - globular clusters: general - globular clusters: individual: NGC 6397 globular clusters: individual: NGC 6752

\section{Introduction}

Although nitrogen is one of the most abundant elements in the cosmos, it has been difficult to obtain significant numbers of $\mathrm{N}$ abundance measurements in metal poor stars, which would enable the $\mathrm{N}$ evolution of the Galaxy to be studied quantitatively (see e.g. Israelian et al. 2004; and references therein). Nitrogen tracks iron $([\mathrm{N} / \mathrm{Fe}] \sim 0)$ down to a metallicity of $[\mathrm{Fe} / \mathrm{H}] \sim-1.0$. The evolution at lower metallicities is less clear: data for many stars appear to indicate that $\mathrm{N}$ continues to track iron, although a few field stars are found to be nitrogen-rich (the prototypes of the class being HD 74000 and HD 160617, Bessell \& Norris 1982), while others seem to show $[\mathrm{N} / \mathrm{Fe}]$ ratios below solar. In their investigation of the chemical composition in the lowest metallicity stars, Spite et al. (2005) suggested that a possible interpretation of the data in the metallicity range $-4.0 \leq[\mathrm{Fe} / \mathrm{H}] \leq-2.5$ is a bimodal distribution of nitrogen abundances, the majority of stars at $[\mathrm{N} / \mathrm{Fe}] \sim+0.4$ and the remainder at $[\mathrm{N} / \mathrm{Fe}] \sim-1.0$. Given the complexity of its nucleosynthesis, iron is not the most suitable choice of reference element to study chemical evolution, as discussed by Cayrel et al. (2004), and an $\alpha$-chain element such as

\footnotetext{
* Based on observations collected at the ESO VLT, Paranal Observatory, Chile, program 075.D-0807(A).
}

$\mathrm{O}$ or $\mathrm{Mg}$ is preferable. The $[\mathrm{N} / \alpha]$ ratio begins to decline as one moves from solar to lower metallicities (see e.g. Fig. 17 of Spite et al. 2005) and at low metallicities it reaches a constant value, a plateau at $[\mathrm{N} / \alpha] \sim-0.9$, although it could be argued that there are in fact two plateaus, the second found around $[\mathrm{N} / \alpha] \sim-1.4$.

From the nucleosynthetic point of view, there is only one mode of nitrogen production: it is formed during the $\mathrm{CNO}$ cycle, from available $\mathrm{C}$ and $\mathrm{O}$. Since this requires the pre-existence of nuclei of $\mathrm{C}$ and $\mathrm{O}$, we expect the production of $\mathrm{N}$ to increase with increasing abundance of $\mathrm{C}$ and $\mathrm{O}$, i.e. with metallicity. Nitrogen would then be a so-called purely secondary element, to distinguish it from primary elements, such as the $\alpha$-chain elements, whose yield is independent of metallicity. The ratio of a purely secondary element to a primary one should be a monotonically increasing function of metallicity. The fact that $[\mathrm{N} / \alpha]$ shows a plateau at low metallicity demonstrates that $\mathrm{N}$ cannot be a purely secondary element. We note that, although the concept of primary and secondary elements is a useful simplification, for majority of the elements the production history is sufficiently complex for either of these terms to be inappropriate.

Although the nucleosynthetic process responsible for the production of nitrogen is unique, its two types of behaviour can only be explained if it was formed in different sites. Intermediate 
mass stars in the AGB phase (IM-AGB stars, Ventura et al. 2002), in which the H-burning shell operates by means of the $\mathrm{CNO}$ cycle, have always been considered major producers of nitrogen. For these stars, the necessary $\mathrm{C}$ and $\mathrm{O}$ nuclei are those already present at the time of formation of the star and the production mode would be secondary. Massive stars are other possible $\mathrm{N}$ producers. In such stars, the central $\mathrm{H}$ burning occurs throughout the $\mathrm{CNO}$ cycle, and again the mode of production would be secondary. However, if the star is rotating, as soon as the central He burning is ignited, fresh $\mathrm{C}$ and $\mathrm{O}$ produced inside the core may be transported by rotational mixing to the $\mathrm{H}$ burning shell. This would then produce $\mathrm{N}$ in a primary fashion, since the necessary parent nuclei are created in situ (see Maeder and Meynet 2005 for a complete discussion). In such a scenario, $\mathrm{N}$ production could not be purely primary, since some secondary production is unavoidable. A primary mode of production of $\mathrm{N}$ may also occur in IM-AGB stars, provided a way of mixing the products of the He burning shell into the $\mathrm{H}$ burning shell exists.

The two above mentioned producers (IM-AGB stars and massive stars) release their nucleosynthetic products, which become available for the next generation of stars, on quite different timescales. Massive stars explode as core-collapse supernovae on timescales shorter that $10 \mathrm{Myr}$, while IM-AGB release their products by means of winds or a planetary nebula phase on a timescale of between $300 \mathrm{Myr}$ and $1 \mathrm{Gyr}$, depending on their mass and metallicity.

The pattern of $\mathrm{N}$ abundances in globular clusters is more complex than in field stars. As for other elements, there is a considerable scatter in the $\mathrm{N}$ abundance measurements of stars in the cluster. From studies conducted with intermediate-band photometry or low-resolution spectra, it has become clear that many clusters display variations in N abundances (see Kraft 1979, for a review of these older results). For many years these effects were supposed to be caused by mixing in giant stars, which were the only stars that could be analysed (see Norris 1981, for an interesting proposal for the role of rotation). Due to the availability of $8 \mathrm{~m}$ class telescopes, these effects can be now investigated to the TO of a few globular clusters, and abundance variations appear to persist. Since a TO star should not experience mixing, another explanation for these variations must be found (Carretta et al. 2005). Although it is generally accepted that the stars we presently observe in GCs have been formed out of material which has been inhomogeneously polluted by a previous generation of stars, there is little consensus on which stars were responsible for the pollution. Proposed candidates are IMAGB stars (D' Antona et al. 2005) and massive stars (see e.g. Decressin et al. 2007a), including Wolf-Rayet objects (Smith 2006).

It appears timely to compare the $\mathrm{N}$ abundance observed in unevolved field and globular cluster stars, by making the effort to place them on the same abundance scale, and to use this comparison to shine some light on the formation of nitrogen in the Galaxy, on the formation process of globular clusters and on the globular clusters - halo relationship.

We present abundances for five TO stars (3 in NGC 6397 and 2 in NGC 6752) derived from the compact band-head of the (00 ) vibrational band of the $\mathrm{NH} A^{3} \Pi-X^{3} \Sigma^{-}$system at $3360 \AA$. This feature is stronger than the UV CN bands used in Carretta et al. (2005) and should provide a more accurate measurement. The $\mathrm{N}$ abundance derived from the NH bands does not depend on either $\mathrm{C}$ nor $\mathrm{O}$; on the opposite, when the $\mathrm{CN}$ bands are used, the derived $\mathrm{N}$ abundance depends on the assumed $\mathrm{C}$ and $\mathrm{O}$ abundances. The $\mathrm{O}$ abundance plays an important role because the majority of $\mathrm{C}$ atoms are locked in the tightly bound $\mathrm{CO}$ molecules. For four out of the five stars studied, preliminary abundances, based on the NH bands, were presented by Pasquini et al. (2004, 2007). However, we deemed that a reanalysis, using the same molecular data and analysis procedure as in Ecuvillon et al. (2004) and Israelian et al. (2004), was necessary to allow an unambiguous comparison of the $\mathrm{N}$ abundances in the GCs with those in field stars of comparable metallicity.

\section{Sample stars and observations}

Being the closest GCs in the southern hemisphere, NGC 6397 and NGC 6752 are amongst the most studied GCs. Many high resolution spectra exist, including some of TO stars.

The objects and the observations presented here were described in Pasquini et al. (2004, 2007). Only star 1406 of NGC 6397 was not contained in the Pasquini et al. (2004) sample, because the $\mathrm{S} / \mathrm{N}$ ratio obtained in the Be region was too low to attempt any Be abundance analysis.

The spectra discussed in this paper are only those acquired with the blue arm of the UVES spectrograph (Dekker et al. 2000) on the ESO Kueyen-VLT 8.2 m telescope. An identical setup was used for the observations of the two globular clusters i.e. central wavelength $3460 \AA, 1^{\prime \prime} 0$ slit and $2 \times 2$ on-chip binning. The effective resolution was around $R \sim 45000$. Further details were provided by Pasquini et al. (2004, 2007).

The two stars of NGC 6752 were selected to correspond to the extremes of the O-Na anti-correlation from Gratton et al. (2001): star 4428 is representative of the O-rich, Li-rich component, while star 200613 is representative of the O-poor - Li-poor component. By observing stars at the extremes of the chemical distribution, we hoped to maximise the probability of observing differences in the $\mathrm{N}$ abundance.

Table 1 summarises the characteristics of the studied stars, including the abundances of the single elements derived from the literature. The $[\mathrm{N} / \mathrm{H}]$ abundance measured in the present work is also provided, in the last column. The stellar parameters listed in Table 1 are those adopted in our spectroscopic analysis. Only abundances of those elements known to vary from star to star $(\mathrm{O}$, $\mathrm{Na}, \mathrm{Li}, \mathrm{N})$ are listed in Table 1 . The reader can find additional element abundances in the following papers: Gratton et al. (2001), James et al. (2004), and Carretta et al. (2005).

\section{Abundance analysis}

The main aim of this paper is to set the cluster stars on the same $\mathrm{N}$ abundance scale as field stars (Israelian et al. 2004; Ecuvillon et al. 2004). We therefore used precisely the same line-fitting method, and molecular and atomic line data as Ecuvillon et al. (2004). The model atmospheres were the same as those used by Pasquini et al. $(2004,2007)$ and were computed with version 9 of the ATLAS code (Kurucz 1993, 2005) and its Linux version (Sbordone et al. 2004; Sbordone 2005). All the models were computed with the "NEW" Opacity Distribution Functions Castelli \& Kurucz (2003), with $1 \mathrm{~km} \mathrm{~s}^{-1}$ micro-turbulence, a mixing-length parameter $\alpha_{\mathrm{MLT}}$ of 1.25 and no overshooting. The synthetic spectra were computed with the line formation code MOOG (Sneden 1973, 1974, 2007).

Figures 1 and 2 show the spectra of the five stars compared with synthetic spectra. The synthetic spectrum with the best-fit $\mathrm{N}$ abundance is always shown as a solid line.

The stellar parameters adopted are given in Table 1. A difference in $T_{\text {eff }}$ of $\pm 100 \mathrm{~K}$ implies a change in $[\mathrm{N} / \mathrm{H}]$ of \pm 0.09 dex. In spite of the differences in atomic and molecular data involved 
Table 1. NGC 6752 sample stars, their atmospheric parameters, and abundances. The atmospheric parameters, $[\mathrm{Fe} / \mathrm{H}],[\mathrm{O} / \mathrm{H}]$ and $[\mathrm{Na} / \mathrm{Fe}]$ are from Gratton et al. (2001); $\log (\mathrm{Li} / \mathrm{H})$ from Pasquini et al. (2005); and [Be/H] from Pasquini et al. (2006); [N/H] from this work.

\begin{tabular}{lllllllll}
\hline \hline Star & $T_{\text {eff }}$ & $\log g$ & {$[\mathrm{Fe} / \mathrm{H}]$} & {$[\mathrm{O} / \mathrm{H}]$} & {$[\mathrm{Na} / \mathrm{Fe}]$} & $\log (\mathrm{Li} / \mathrm{H})$ & $\log (\mathrm{Be} / \mathrm{H})$ & {$[\mathrm{N} / \mathrm{H}]$} \\
\hline & & & & & & & & \\
4428 & 6226 & 4.28 & -1.52 & -1.28 & -0.35 & 2.50 & -12.04 & $-1.2 \pm 0.1$ \\
200613 & 6226 & 4.28 & -1.56 & $/$ & 0.64 & 2.13 & $<-12.2$ & $0 \pm 0.1$ \\
\hline
\end{tabular}

Table 2. NGC 6397 stars, their atmospheric parameters and abundances. The atmospheric parameters, are from Bonifacio et al. (2002), [Be/H] from Pasquini et al. (2004), [N/H] from this work.

\begin{tabular}{lllllllll}
\hline \hline Star & $T_{\text {eff }}$ & $\log g$ & {$[\mathrm{Fe} / \mathrm{H}]$} & {$[\mathrm{O} / \mathrm{H}]$} & {$[\mathrm{Na} / \mathrm{Fe}]$} & $\log (\mathrm{Li} / \mathrm{H})$ & $\log (\mathrm{Be} / \mathrm{H})$ & {$[\mathrm{N} / \mathrm{H}]$} \\
\hline & & & & & & & & \\
2111 & 6207 & 4.1 & -2.01 & -2.24 & +0.17 & 2.33 & -12.27 & $-0.76 \pm 0.1$ \\
228 & 6274 & 4.1 & -2.05 & -1.64 & +0.16 & 2.28 & -12.43 & $-0.75 \pm 0.15$ \\
1406 & 6345 & 4.1 & -2.04 & $/$ & $/$ & 2.37 & $/$ & $-1 \pm 0.2$ \\
\hline
\end{tabular}

and of the line formation code used, the comparison between the present results and those of Pasquini et al. (2004, 2007) is excellent (with agreement to within $0.05 \mathrm{dex}$ ), apart from star 4428 of NGC 6752, for which there is a discrepancy of a factor of two. In spite of this discrepancy, we confirm the huge abundance difference ( $1.3 \mathrm{dex}$ ) between the two observed stars of this cluster.

Carretta et al. (2005) used the CN band at $3880 \AA$ in their analysis to derive $\mathrm{N}$ in several stars in GCs. For all the dwarfs in NGC 6397 they found only upper limits, which were higher than, and therefore consistent with, our measurements. As far as the NGC 6752 dwarfs are concerned, they quote a value similar to ours for star 200613, but they quote a value 10 times higher than ours for star 4428, which should instead be considered as an upper limit (Carretta, private communication; see also Pasquini et al. 2007).

\section{Discussion}

\subsection{Comparison with field stars}

Our N abundances for GC stars can be consistently compared with results for field stars from Ecuvillon et al. (2004) and Israelian et al. (2004).

Figure 3 shows a plot of $[\mathrm{N} / \mathrm{H}]$ abundance versus $[\mathrm{Fe} / \mathrm{H}]$ for field and cluster stars. Most field stars closely follow a 1:1 relationship that has a scatter compatible with the error measurements (quoted as $\sim \pm 0.15$ by Israelian et al. 2004, and given for reference in the upper left corner of the figure). The [N/O] ratio is shown in Fig. 4. In the present discussion we ignore any complications due to the $\mathrm{O}$ abundances displayed being derived in an inhomogeneous way using different $\mathrm{O}$ indicators. We instead accept all measurements with the caveat that any systematic differences in measurement derived by different methods would tend to blur any existing trends. Two points are clearly evident in Fig. 2:

1. in general, the cluster stars do not show the same properties of the field stars;

2. the two clusters do not show the same behaviour.

The first point is in part expected, since we have known for a long time of the presence of chemical anomalies in GC stars, which includes the presence of $\mathrm{N}$ rich stars. Alhough N-rich stars are known in the field, they are extremely rare, and a typical sample, such as that of Israelian et al. (2004), contains at most one such object.

The two NGC 6752 stars show a large spread in N (almost a factor of 20) and the cluster stars, including all stars observed in
NGC 6397, have a high N abundance, departing from the field stars relationship significantly. This is clearly related to the peculiar formation of GCs, and we will return to this point in the next section.

As far as the second point is concerned, we believe that it should be considered carefully, because it has not been well studied to date. For this reason, we consider the two clusters separately.

NGC 6752 is a cluster showing a wide range of chemical inhomogeneities and anti-correlations. But, in its anomalies, the observed trends are extremely consistent. The difference of a factor of approximately 20 in $\mathrm{N}$ among the two stars is high, but is in line with the other significant differences recorded for both $\mathrm{Na}$ (factor 10) and $\mathrm{Li}$ (factor 2.5) in these stars and in oxygen (factor 4) in other stars, which have an abundance pattern similar to star 200613 (in 200613, O was not measured).

With our new value, the $\mathrm{N}$ of star 4428 is higher, but formally compatible with the field stars having similar metallicity. Even if we do not know how this GC formed, the observations are compatible with the idea that star 4428 is the prototype of an "unpolluted" star, similar to its field counterparts of almost identical $[\mathrm{Fe} / \mathrm{H}]$, while star 200613 is, in contrast, the prototype of a highly polluted star, with high $\mathrm{N}$, high $\mathrm{Na}$, low $\mathrm{Li}$, and low $\mathrm{O}$, showing therefore the signature of gas which experienced high temperatures where nuclear cycles such as $\mathrm{CNO}, \mathrm{Ne}-\mathrm{Na}$, and the consequent Li destruction, have occurred.

When plotting $[\mathrm{N} / \mathrm{H}]$ versus $[\mathrm{O} / \mathrm{H}]$ (Fig. 4 ), the situation is similar, but we have $[\mathrm{O} / \mathrm{H}]$ abundances for only 3 out of five stars. The impression is that the cluster stars (including star 4428) do not follow the relationship defined by the field stars. This may be because star 4428 has an $[\mathrm{O} / \mathrm{Fe}]$ ratio that is lower, by roughly a factor of 2 , than field stars of the same $[\mathrm{Fe} / \mathrm{H}]$ : when $[\mathrm{N} / \mathrm{H}]$ is plotted against $[\mathrm{Fe} / \mathrm{H}]$, star 4428 follows the trend of field stars, but when plotted versus $[\mathrm{O} / \mathrm{H}]$ it appears to have too much nitrogen, with respect to field stars of the same $[\mathrm{O} / \mathrm{H}]$. Clearly in a cluster such as NGC 6752, which has a strong Na-O anticorrelation, it is difficult to identify the "unpolluted" oxygen content of the cluster. Among the stars analysed by Gratton et al. (2001), there is one dwarf and two subgiants that have higher $[\mathrm{O} / \mathrm{H}]$ than star 4428 . We chose star 4428 to be a template of the "unpolluted" population, because it is the star of the lowest $[\mathrm{Na} / \mathrm{Fe}]$. New measurements of $\mathrm{N}$, based on the $\mathrm{NH}$ bands for all the stars observed by Gratton et al. (2001), would be desirable, given the weakness of the $\mathrm{CN}$ bands and the difficulty in using them to measure $\mathrm{N}$. 

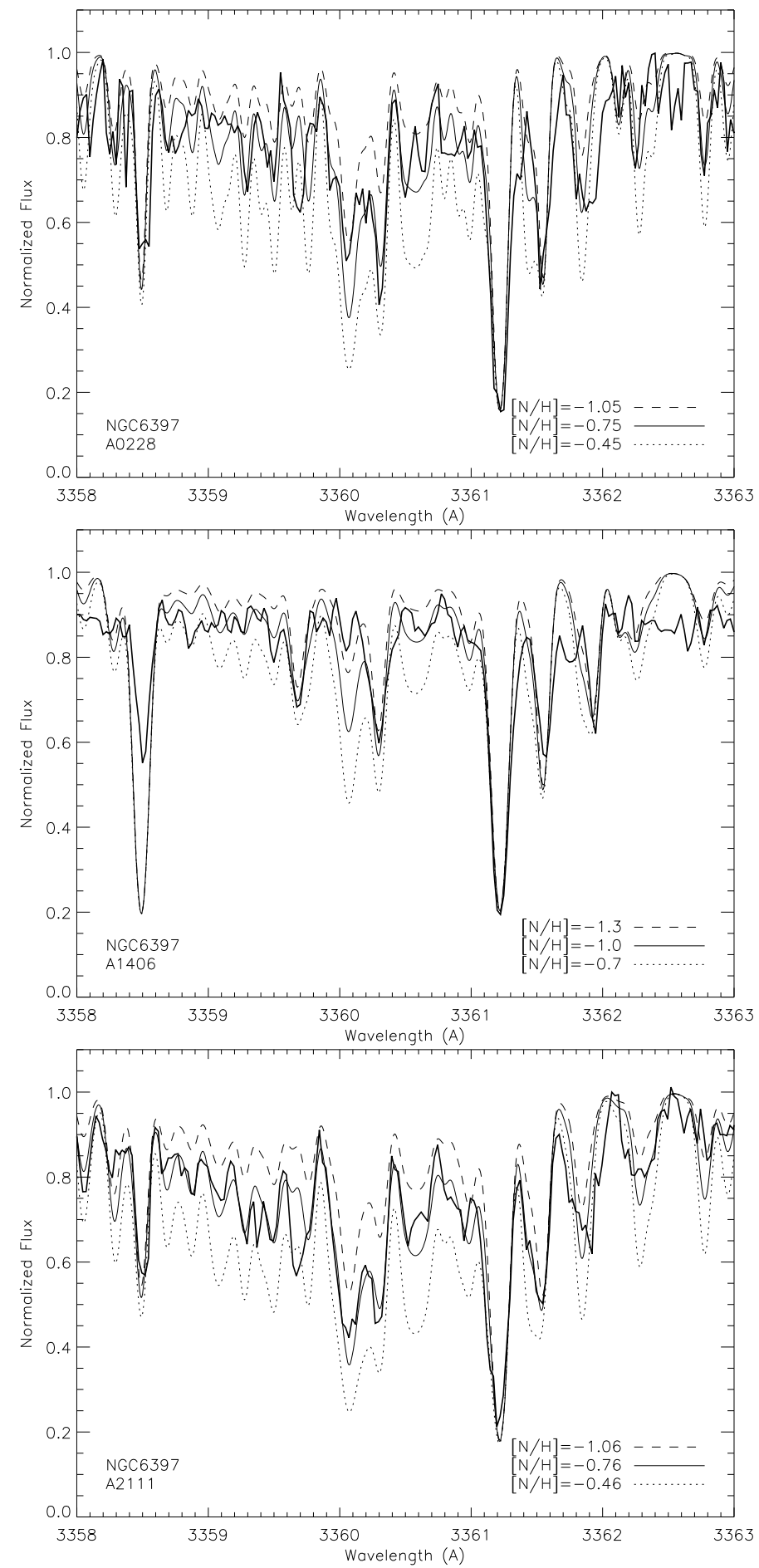

Fig. 1. UVES spectra of the NGC 6397 TO stars in the NH band. best fit models are shown in bold, while models varying by \pm 0.3 dex in $\mathrm{NH}$ are shown as dashed and dotted lines.

In summary, our results for NGC 6752 confirm that the chemical composition of stars is consistent with assuming that each object is composed of a mixture of "pristine" and "processed" material. The "pristine" material is possibly $\mathrm{N}$ rich, but otherwise indistinguishable from the composition of typical halo stars. The "processed" material is $\mathrm{N}$ and Na rich, $\mathrm{O}$ and Li poor.

On the other hand the NGC 6397 cluster stars are more intriguing: the NGC 6397 objects have similar N abundances, with a negligible spread, but at an absolute level about 10 times higher than field stars of similar metallicity. It is impossible, even
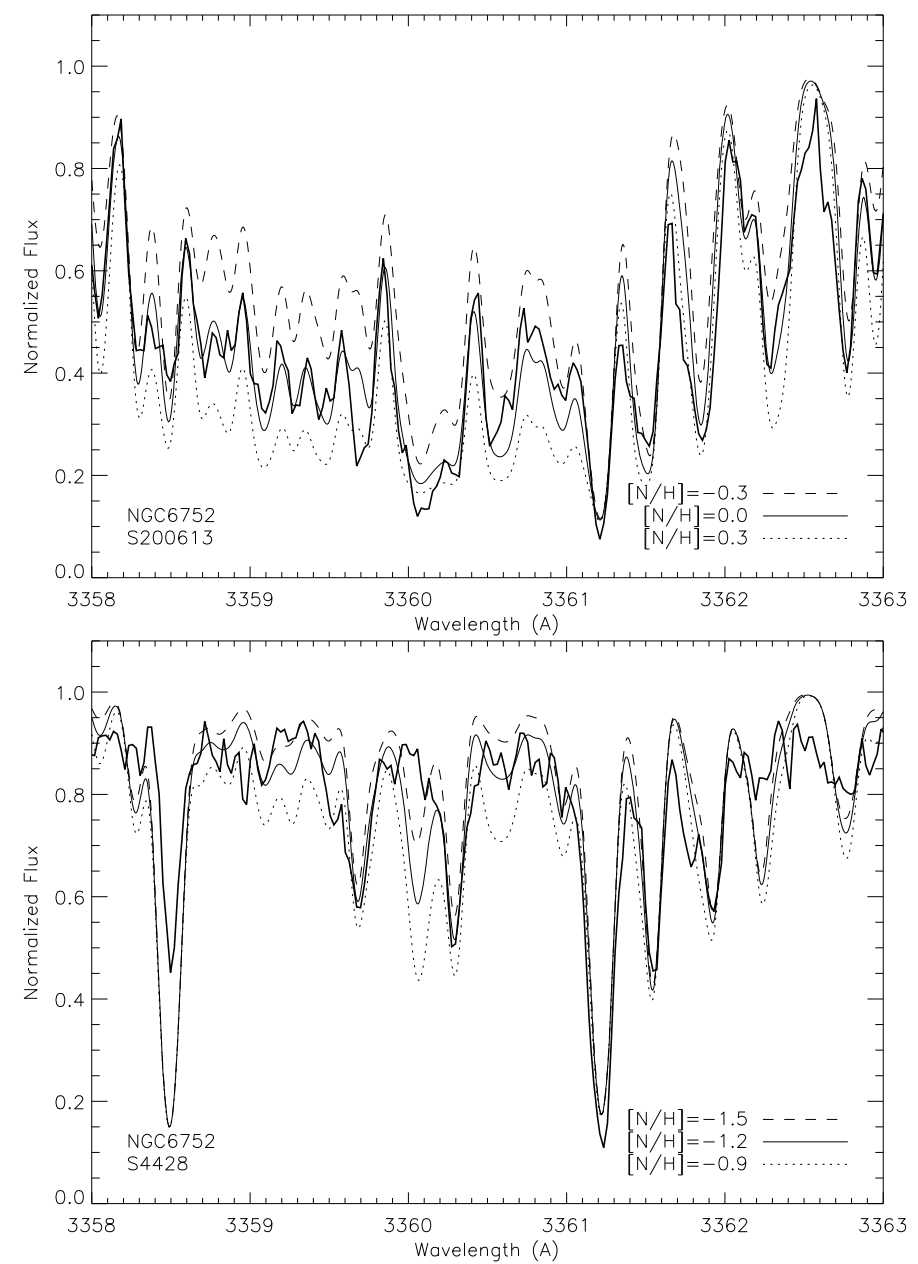

Fig. 2. UVES spectra of the NGC 6752 TO stars in the NH band. best fit models are shown in bold, while models varying by \pm 0.3 dex in $\mathrm{NH}$ are shown as dashed and dotted lines.

assuming the highest $\mathrm{N}$ production rates predicted for rapidly rotating massive stars, to account for such a large amount of N production in a standard Galactic chemical evolutionary model (see e.g. Chiappini et al. 2006): such a high $\mathrm{N}$ abundance is therefore the direct proof that the NGC 6397 stars have been affected by a peculiar process. In their extensive study of the globular cluster system of M 31 Burstein et al. (2004), using integrated light spectra, demonstrated that the older clusters of $M 31$ have considerably stronger $\mathrm{NH}$ bands than Galactic clusters. The reasons for this difference is unclear. NGC 6397 was not among the Galactic globular clusters studied by Burstein et al. (2004), but adopting the naive point of view that $\mathrm{NH}$ band strength is related to $\mathrm{N}$ abundance, one may expect that, in integrated light, NGC 6397 should show a stronger NH band than other Galactic globular clusters, although not necessarily as strong as that of the M 31 clusters. It remains to be investigated if the mechanism that causes a strong NH band in the globular clusters of M 31 is the same, or akin to, that producing the high $\mathrm{N}$ abundance in NGC 6397.

In contrast the oxygen content of the cluster is low, that is at least a factor of two lower than field stars of similar metallicity. Even if the behaviour of oxygen in metal poor stars is highly debated, oxygen has been measured at the level of only $[\mathrm{O} / \mathrm{Fe}]=0.2-0.3$ in NGC 6397 by a number of independent studies (Thévenin et al. 2001; Pasquini et al. 2004; Gratton et al. 2001). Carretta et al. (2005) show that the C in NGC 6397 is 


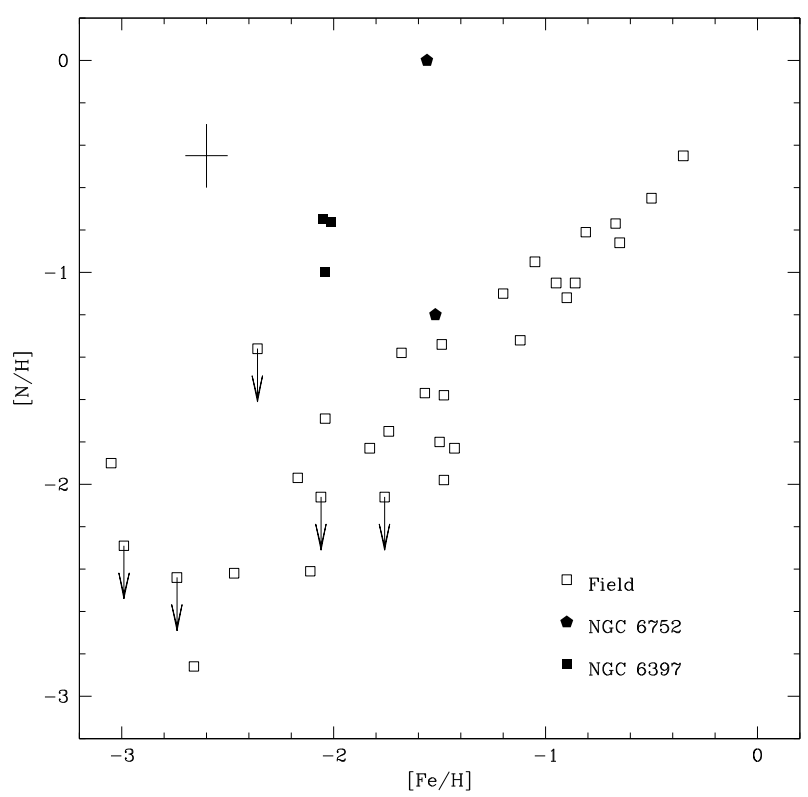

Fig. 3. $[\mathrm{N} / \mathrm{H}]$ versus $[\mathrm{Fe} / \mathrm{H}]$ for the field stars of Israelian et al. (2004) and the TO stars of the two globular clusters NGC 6397 and NGC 6752 (this paper). A typical error bar is indicated at the top left corner.

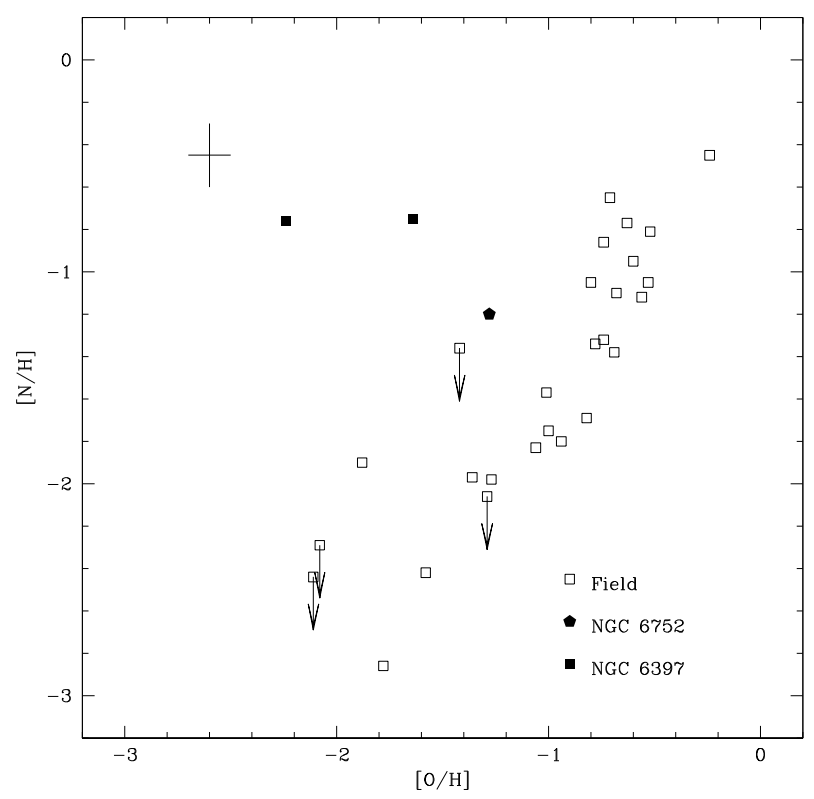

Fig. 4. $[\mathrm{N} / \mathrm{H}]$ versus $[\mathrm{O} / \mathrm{H}]$ for the field stars of Israelian et al. (2004) and the TO stars of the two globular clusters NGC 6397 and NGC 6752 (this paper). A typical error bar is indicated at the top left corner.

at a level of $[\mathrm{C} / \mathrm{Fe}] \sim 0$, which is similar to that measured for field stars of similar metallicity. This indicates that, for dwarfs observed in this cluster, the entire $\mathrm{CNO}$ balance is different from that of field stars. On the other hand, the Li abundance (Molaro \& Pasquini 1994; Pasquini \& Molaro 1996; Thévenin et al. 2001; Bonifacio et al. 2002; Korn et al. 2006) is absolutely consistent with the Spite plateau (Spite \& Spite 1982a,b), and Na, with a slightly supersolar value, does not vary at a significant level in stars observed so far (Gratton et al. 2001).

As far as the chemical composition of TO stars is concerned, the homogeneity in NGC 6397 stars appears far higher than in NGC 6752, in that only a significant variation in oxygen was reported by Pasquini et al. (2004) for two stars in this sample, while no substantial spread is observed in any other element among the main sequence stars. Only three subgiants show evidence for strong $\mathrm{Na}$ and $\mathrm{N}$ differences and no real "anticorrelation" can be deduced by abundances so far published (Gratton et al. 2001; Carretta et al. 2005, Table 1).

Of course we must be careful in deriving strong conclusions from such a low number of measurements, and we are aware that some previous trends could be simply the result of a combination of a limited sample and significant errors. However, we summarise this part of the discussion by stating that, apart from some similarities, the two clusters follow different trends of $\mathrm{N}$ abundance and, in general, variation in light element abundances. While the abundances of the stars of NGC 6752 inidicate evidences of pollution, the NGC 6397 stars show signatures of $\mathrm{CNO}$ (and probably NeNa) processed material, but no clear anticorrelation, a far more significant homogeneity in abundances, and a Li content similar to that of the Spite plateau.

\subsection{About $G C$ formation}

One fundamental consideration in the discussion of the formation scenarios is the fact that anti-correlations persist in evolved stars after the dredge-up (see e.g. Grundhal et al. 2002). This shows that the entire stellar mass, and not only the external stellar layers, is affected by the chemical anomalies. As far as this paper is concerned, we summarise the ongoing discussion on the formation of $\mathrm{GC}$ by two main points:

- The mass of the "polluting" stars: some authors propose that the CNO cycling is generated by intermediate mass AGB stars (Ventura et al. 2002; D' Antona et al. 2005), while others favour massive rotating stars (Maeder \& Meynet 2002; Decressin et al. 2007b). Each of these schemes has strong and weak points. In an attempt to summarise them, we can say that IM-AGB stars have the advantage of being "natural" $\mathrm{N}$ producers, able to also produce $\mathrm{Li}$ (and explain the behaviour of NGC 6397 by means of the so-called CameronFowler mechanism; Cameron \& Fowler 1971), but having at present two main problems: the difficulty in reproducing the observed yields of other elements (in particular $\mathrm{Na}$ ), the fact that for NGC 6397 a natural conspiracy should have produced an ad hoc amount of Li, precisely the same as observed for the Spite plateau (Bonifacio et al. 2002). Finally, the IM-AGB hypothesis would require a peculiar IMF, heavily flat-topped (Prantzos \& Charbonnel 2005; Chiappini et al. 2006). As far as the rotating massive star hypothesis is concerned, this scheme mitigates the IMF requirements, and can more successfully explain some of the observed yields, although even the most extreme models cannot account for the observed high range of the $\mathrm{Mg}$ - $\mathrm{Al}$ correlation, unless some of the employed nuclear cross-sections are affected by large errors (Decressin et al. 2007b).

One important conclusion of both hypotheses is that the "polluted" stars should have a far higher ${ }^{4} \mathrm{He}$ abundance. Higher ${ }^{4} \mathrm{He}$ abundance is claimed to be observed in one of the Main Sequences of $\Omega$ Cen Piotto et al. (2005) and of NGC 2808 Piotto et al. (2007); it would naturally explain the blue extension of HB in NGC 6752 and other clusters (D'Antona et al. 2005). On the other hand, a substantially higher ${ }^{4} \mathrm{He}$ abundance should correspond spectroscopically to an apparent higher abundance of heavier elements and higher spectroscopic gravity in ${ }^{4} \mathrm{He}$ rich stars (Böhm-Vitense 1979). A spread in He abundances should produce a spread in the abundances of all elements, including iron-peak elements. The results of Gratton et al. (2001) in NGC 6397 and 
NGC 6752 exclude any significant spread in Fe abundances in either cluster.

- The second open issue is related to the overall mass of the structures containing the contaminants. Most analyses have considered to date self-pollution, that is, where all polluted material was created within the cluster. New schemes consider the possibility of infall from the exterior (e.g. Bekki et al. 2007). In such schemes, for instance, GCs are the remnants of the core of much more massive dwarf galaxies, receiving processed gas from the entire galaxy, rather than from its own stars only. This scheme would remove the need for an anomalous IMF, but widens the possible parameter space; this is because a new variable, the infall of material, is now included, which has not, however, been considered in detail by chemical evolution models.

We finally recall that most studies attempt to explain all data observed in the different GC within a unique scheme. This is, of course, an ambitious and important approach, but we emphasize that the observational data appear to indicate that different clusters most likely have different evolutionary histories and any attempt to describe all observations with a unique model might be premature.

The measured abundances enable the composition of the polluting gas to be studied in some detail. For NGC 6752, we can safely assume that the two stars observed are at the extremes of the chemical distribution for stars in the cluster.

To analyse pollution, $\mathrm{Li}$ is the best element to consider, since it is destroyed at temperatures at which CNO cycling occurs. The difference in $\mathrm{Li}$ between the two stars is a factor of about 2.5. If no Li production has occurred, this implies a fraction of $60 \%$ of processed material in the contaminated star. The observed $\mathrm{N}$ enhancement would imply a 25 -fold $\mathrm{N}$ production in the polluting gas. This can be assumed to be a solid lower limit to the yield, in the case for which no infall is present. If the polluting gas is rich in $\mathrm{Li}$, the fraction of polluting gas will be higher than $60 \%$ and, as a consequence, the overproduction of $\mathrm{N}$ in the previous generation will be lower than estimated above. We note that a 25 -fold $\mathrm{N}$ production reach the upper limit of present models of massive stars. Detailed predictions of the $\mathrm{N}$ production rates were computed e.g. by Decressin et al. (2007b), who found that massive stars could generate up to $[\mathrm{N} / \mathrm{Fe}] \sim 1.5$ in their winds, or a factor of 30 at most.

The constraints provided by oxygen are also important: the ratio in the $\mathrm{O}$ abundance of star 4428 (O-rich) to that of the most O-poor stars observed in NGC 6752 is about 4.5 (Carretta et al. 2005), which would place a lower limit constraint of $22.5 \%$ on the amount of pristine material, in case the processed material had no $\mathrm{O}$ at all.

The NGC 6752 data are consistent with a picture in which the stars in this cluster are formed by a mixture of "pristine" and "processed" material. The maximum observed "pollution" amounts to about $60 \%$ of the gas forming the stars. This would imply a polluting material with no $\mathrm{Li}$, no $\mathrm{O}$, and $\mathrm{N}$ enhanced by 25 times (and Na by a similar amount). In a closed system it may be difficult to produce the high amount of $\mathrm{N}$ required by this scenario. The variations in oxygen abundance measured among the extreme stars suggest an even higher level of polluted material, which would require some Li production, and in this case the $\mathrm{N}$ (and $\mathrm{Na}$ ) overabundance should be proportionally lower in the polluting gas.
The abundances of NGC 6397 cannot be accounted for by a simple pollution model, since the measured variations in oxygen are not accompanied by similar variations in Li (Bonifacio et al. 2002) and in $\mathrm{N}$ (this paper).

Acknowledgements. P.B. acknowledges financial support from EU contract MEXT-CT-2004-014265 (CIFIST).

\section{References}

Bekki, K., Campbell, S. W., Lattanzio, J. C., \& Norris, J. E. 2007, MNRAS, 377, 335

Bessell, M. S., \& Norris, J. 1982, ApJ, 263, L29

Böhm-Vitense, E. 1979, ApJ, 234, 521

Bonifacio, P., Pasquini, L., Spite, F., et al. 2002, A\&A, 390, 91

Burstein, D., David, Li, Yong, Freeman, K. C., et al. 2004, ApJ, 614, 158

Cameron, A. G. W., \& Fowler, W. A. 1971, ApJ, 164, 111

Carbon, D. F., Barbuy, B., Kraft, R. P., Friel, E. D., \& Suntzeff, N. B. 1987, PASP, 99, 335

Carretta, E., Gratton, R. G., Lucatello, S., Bragaglia, A., \& Bonifacio, P. 2005, A\&A, 433, 597

Castelli, F., \& Kurucz, R. L. 2003, in IAU Symp., ed. N. Piskunov, W. W. Weiss, \& D. F. Gray, 20P

Charbonnel, C., \& Prantzos, N. 2006, astro-ph 6220

Cayrel, R., Depagne, E., Spite, M., et al. 2004, A\&A, 416, 1117

Chiappini, C., et al. 2006, in Nuclei in in the Cosmos IX, 80.1

D’Antona, C., Bellazzini, M., Caloi, V., et al. 2005, ApJ, 631, 868

Decressin, T., Meynet, G., Charbonnel, C., Prantzos, N., \& Ekström, S. 2007a, A\&A, 464, 1029

Decressin, T., Charbonnel, C., \& Meynet, G. 2007b, A\&A, 475, 859

Dekker, H., D' Odorico, S., Kaufer, A., Delabre, B., \& Kotzlowski, H. 2000, SPIE, 4008, 534

Ecuvillon, A., Israelian, G., Santos, N. C., et al. 2004, A\&A, 418, 703

Gratton, R. G., Bonifacio, P., Bragaglia, A., et al. 2001, A\&A, 369, 87 (G01)

Grundahl, F., Briley, M., Nissen, P. E., \& Feltzing, S., et al. 2002, A\&A, 385, L84

Israelian, G., Ecuvillon, A., Rebolo, R., et al. 2004, A\&A, 421, 649

Kraft, R. P. 1979, ARA\&A, 17, 309

Korn, A. J., Grundahl, F., Richard, O., et al. 2006, Nature, 442, 657

Kurucz, R. 1993, ATLAS9 Stellar Atmosphere Programs and $2 \mathrm{~km} / \mathrm{s}$ grid. Kurucz CD-ROM No. 13, Cambridge, Mass.: Smithsonian Astrophysical Observatory

Kurucz, R. L. 2005, Mem. Soc. Astron. Ital. Supp., 8, 14

James, G. , Francois, P., Bonifacio, P., et al. 2004, A\&A, 414, 1071

Maeder, A., \& Meynet, G. 2005, A\&A, 440, 105

Meynet, G., \& Maeder, A. 2002, A\&A, 390, 561

Molaro, P., \& Pasquini, L. 1994, A\&A, 281, L77

Norris, J. 1981, ApJ, 248, 177

Pasquini, L., \& Molaro, P. 1996, A\&A, 307, 761

Pasquini, L., Bonifacio, P., Randich, S., et al. 2004, A\&A, 426, 651

Pasquini, L., Bonifacio, P., Molaro, P., et al. 2005, A\&A, 441, 549

Pasquini, L. Bonifacio, P., Randich, S., et al. 2007, A\&A, 464, 601

Piotto, G., Villanova, S., Bedin, L. R., et al. 2005, ApJ, 621, 777

Piotto, G., Bedin, L. R., Anderson, J., et al. 2007, ApJ, 661, L53

Prantzos, N., \& Charbonnel, C. 2006, A\&A, 458, 135

Sbordone, L. 2005, Mem. Soc. Astron. Ital. Supp., 8, 61

Sbordone, L., Bonifacio, P., Castelli, F., \& Kurucz, R. L. 2004, Mem. Soc. Astron. Ital. Suppl., 5, 93

Smith, G. H. 2006, PASP, 118, 1225

Sneden, C. A. 1973, Ph.D. Thesis

Sneden, C. 1974, ApJ, 189, 493

Sneden, C. 2007, http://verdi.as.utexas.edu/moog.html

Spite, M., \& Spite, F. 1982a, Nature, 297, 483

Spite, F., \& Spite, M. 1982b, A\&A, 115, 357

Spite, M., Cayrel, R., Cayrel, R., Plez, B., et al. 2005, A\&A 430, 655

Thévenin, F., Charbonnel, C., de Freitas Pacheco, J. A., et al. 2001, A\&A, 373, 905

Ventura, P., D’Antona, F., \& Mazzitelli, I. 2002, A\&A, 393, 215 